\title{
Decidable Controller Synthesis for Classes of Linear Systems
}

\author{
Omid Shakernia ${ }^{1}$, George J. Pappas ${ }^{1,2}$, and Shankar Sastry ${ }^{1}$ \\ 1 Department of EECS, University of California at Berkeley, Berkeley, CA 94704, \\ \{omids, gpappas, sastry\}@eecs . berkeley.edu \\ 2 Department of CIS, University of Pennsylvania, Philadelphia, PA 19104 \\ pappasg@grip.cis.upenn.edu
}

\begin{abstract}
A problem of great interest in the control of hybrid systems is the design of least restrictive controllers for reachability specifications. Controller design typically uses game theoretic methods which compute the region of the state space for which there exists a control such that for all disturbances, an unsafe set is not reached. In general, the computation of the controllers requires the steady state solution of a Hamilton-JacobiIsaacs partial differential equation which is very difficult to compute, if it exists. In this paper, we show that for classes of linear systems, the controller synthesis problem is decidable: There exists a computational algorithm which, after a finite number of steps, will exactly compute the least restrictive controller. This result is achieved by a very interesting interaction of results from mathematical logic and optimal control.
\end{abstract}

\section{Introduction}

Reachability specifications for hybrid systems require the trajectories of a hybrid system to avoid an undesirable region of the state space. One of the most important problems in the control of hybrid systems is the design of least restrictive controllers which satisfy the reachability specifications. This problem has been considered in the context of classical discrete automata [3]15], timed automata [1], linear hybrid automata 18], and general hybrid systems [12. The framework presented in [12] has been applied to automated vehicles [11], and air traffic management systems [16].

Designing least restrictive controllers for reachability specifications requires computing the set of all initial states for which there exists a control such that for all disturbances, the system will avoid the undesirable region. The least restrictive controller is then a static feedback controller which allows any control value outside this set of initial conditions while allowing all safe control values on the boundary of this set.

The computation of the safe set of initial states for general hybrid systems leads to game theoretic methods, and in particular to the steady state solution to Hamilton-Jacobi-Isaacs equations [12. In general, these partial differential equations are very difficult to solve. In addition, steady state solutions, if they 
exist, may be discontinuous even if the initial problem data is continuous. This is due to the appearance of shocks, and switchings in the optimal control policy.

The above difficulties in the computation of least restrictive controllers naturally raise the following question : Can we find classes of systems where the game theoretic approach does not require the solution of the Hamilton-JacobiIsaacs equation? In this paper, we give a positive answer to the above question for normal linear control systems where the system matrix is either nilpotent or diagonalizable with purely real rational eigenvalues, and with reachability specifications defined by polynomial inequalities. The normality condition requires controllability of the linear system with each input and disturbance. This condition ensures that the optimal control and disturbance are well defined, and unique. For the case of real eigenvalues, normality also ensures that the optimal control and disturbance have a finite number of switchings [13].

Our framework first applies Pontryagin's maximum principle to synthesize the optimal control and worst disturbance. The switching behavior of the control and the disturbance is then abstracted by a hybrid system, on which we perform reachability computations. By combining the recent decidability results of [8.9, with the normality condition which guarantees finite number of switchings [13], we show that the least restrictive controller can be decidably computed. This interesting interplay of results from mathematical logic and optimal control presents us with the first decidable controller synthesis problem for classes of linear systems.

\section{Controller Synthesis Methodology}

In this section, we briefly review the least restrictive controller synthesis methodology for dynamical systems as presented in [12]. Consider the dynamical system

$$
\dot{x}=f(x, u, d)
$$

with state $x \in \mathbb{R}^{n}$, controls $u \in U \subset \mathbb{R}^{n_{u}}$, disturbances $d \in D \subset \mathbb{R}^{n_{d}}$. Suppose there is a target set $G \subset \mathbb{R}^{n}$ which specifies an undesirable region of the state space. In the context of dynamic pursuit-evasion games [210], the goal of the disturbance is to capture the state by driving it into the target set, while the goal of the controller is to remain in the safe set $G^{c}$, the complement of $G$. The target set is described by $G=\left\{x \in \mathbb{R}^{n} \mid h(x)<0\right\}$, for a smooth function $h: \mathbb{R}^{n} \rightarrow \mathbb{R}$.

Let $\mathcal{U}, \mathcal{D}$ be the set of piecewise continuous functions from $\mathbb{R}$ into $U$ and $D$ respectively. Given an initial condition $x_{0} \in \mathbb{R}^{n}$, input $u(\cdot) \in \mathcal{U}$, and disturbance $d(\cdot) \in \mathcal{D}$, the flow of the differential equation (1) is a map $\Phi: \mathbb{R}^{n} \times \mathcal{U} \times \mathcal{D} \times \mathbb{R} \rightarrow \mathbb{R}^{n}$ given by

$$
\Phi\left(x_{0}, u(\cdot), d(\cdot), t\right)=x_{0}+\int_{0}^{t} f(x(\tau), u(\tau), d(\tau)) d \tau
$$


Clearly, the largest set of safe initial states for which the controller can avoid being captured regardless of the disturbance is given by

$$
W=\left\{x_{0} \in \mathbb{R}^{n} \mid \exists u(\cdot) \in \mathcal{U} \forall d(\cdot) \in \mathcal{D} \forall t \geq 0: \Phi\left(x_{0}, u(\cdot), d(\cdot), t\right) \in G^{c}\right\} .
$$

The set $W$ is called the maximal controlled invariant subset of the safe set $G^{c}$. In the differential games literature, $W$ is called the escape set, since there exists a control policy such that the controller can avoid the target set, and $W^{c}$ is called the capture set. While equation (3) conceptually describes the escape set, it hardly affords a method of computing it. However, the capturability requirement can be encoded by a value function $J: \mathbb{R}^{n} \times \mathcal{U} \times \mathcal{D} \times \mathbb{R}_{-} \rightarrow \mathbb{R}$, which, given an initial state $x_{0} \in \mathbb{R}^{n}, u(\cdot) \in \mathcal{U}, d(\cdot) \in \mathcal{D}$ and $t \leq 0$, returns

$$
J\left(x_{0}, u(\cdot), d(\cdot), t\right)=h(x(0)) .
$$

Therefore, the value function is the cost of a trajectory that starts at initial state $x_{0}$ at time $t \leq 0$ and evolves according to system equation (11) with input $u(\cdot)$, disturbance $d(\cdot)$, and ends at final state $x(0)$ at time $t=0$. Since the control tries to avoid $G$ while the disturbance tries to steer the system to $G$, we naturally arrive at the dynamic game

$$
J^{*}\left(x_{0}, t\right)=\max _{u \in \mathcal{U}} \min _{d \in \mathcal{D}} J\left(x_{0}, u(\cdot), d(\cdot), t\right) .
$$

$J^{*}$ is called the optimal value function, since it is the value function corresponding to the optimal controls and disturbances of the dynamic game. The maximal controlled invariant subset of the safe set is described in terms of the optimal value function by

$$
W=\left\{x \in \mathbb{R}^{n} \quad \mid \min _{t \leq 0} J^{*}(x, t) \geq 0\right\}
$$

In order to compute $J^{*}(x, t)$, we first introduce the Hamiltonian

$$
H(x, p, u, d)=p^{T} f(x, u, d),
$$

where $p \in \mathbb{R}^{n}$ is called the co-state. The optimal Hamiltonian is given by

$$
H^{*}(x, p)=\max _{u \in U} \min _{d \in D} H(x, p, u, d) .
$$

The computation of $J^{*}(x, t)$ requires the solution of a modified Hamilton-JacobiIsaacs partial differential equation 12

$$
\begin{aligned}
J^{*}(x, 0) & =h(x) \\
-\frac{\partial J^{*}(x, t)}{\partial t} & =\min \left\{0, H^{*}\left(x, \frac{\partial J^{*}(x, t)}{\partial x}\right)\right\} .
\end{aligned}
$$

Assuming that (7) has a differentiable solution that converges to a function $J_{1}^{*}(x)$ as $t \rightarrow-\infty$, then the set

$$
W=\left\{x \in \mathbb{R}^{n} \mid J_{1}^{*}(x) \geq 0\right\}
$$


is the maximal controlled invariant subset of the safe set $G^{c}$, and the controller $g: \mathbb{R}^{n} \rightarrow 2^{U}$ defined by

$$
g(x)= \begin{cases}\left\{u \in U \mid \min _{d \in D}\left(\frac{\partial J_{1}^{*}(x)}{\partial x}\right)^{T} f(x, u, d) \geq 0\right\} & \text { if } x \in \partial W \\ U & \text { if } x \in W^{o} \cup W^{c}\end{cases}
$$

is least restrictive controller which renders $W$ invariant [12. The controller (9) is least restrictive in the sense that if $g^{\prime}: \mathbb{R}^{n} \rightarrow 2^{U}$ is any other controller that renders $W$ invariant, then $\forall x \in \mathbb{R}^{n}$ we have $g \prime(x) \subseteq g(x)$.

The main difficulty in the above framework is the computation of $W$. In general, solving the Hamilton-Jacobi-Isaacs equation (7) seems necessary for exactly computing $W$. However, there are very difficult issues that must be resolved in this case:

1. Existence and uniqueness of solutions,

2. Existence and uniqueness of steady state solutions,

3. Shocks: non-smooth solutions to smooth problems,

4. Convergence of numerical algorithms.

Given the above difficulties, a natural direction of research is to find classes of systems for which some (or all) of these issues are resolved. In this paper, we adopt this point of view and we will prove the following theorem.

Theorem 1 (Decidable Controller Synthesis). Consider the controller synthesis problem for the dynamical system

$$
\dot{x}=A x+B u+E d
$$

with controls $u \in U \subset \mathbb{R}^{n_{u}}$, disturbances $d \in D \subset \mathbb{R}^{n_{d}}$ and target set $G \subset \mathbb{R}^{n}$ given by

$$
G=\left\{x \in \mathbb{R}^{n} \mid h(x)<0\right\} .
$$

Suppose the dynamical system and target set satisfy the following properties:

1. $A \in \mathbb{Q}^{n \times n}, B \in \mathbb{Q}^{n \times n_{u}}, E \in \mathbb{Q}^{n \times n_{d}}$,

2. For each column $b_{i}$ of $B$, the pair $\left(A, b_{i}\right)$ is completely controllable,

3. For each column $e_{i}$ of $E$, the pair $\left(A, e_{i}\right)$ is completely controllable,

4. The feasible sets of controls $U$ and disturbances $D$ are compact rectangles with rational vertices, that is $U=\prod_{i=1}^{n_{u}}\left[\underline{U}_{i}, \bar{U}_{i}\right]$ and $D=\prod_{i=1}^{n_{d}}\left[\underline{D}_{i}, \bar{D}_{i}\right]$

5. $h \in \mathbb{Q}\left[x_{1}, x_{2}, \ldots, x_{n}\right]$ and $\frac{\partial h}{\partial x}(x) \neq 0$ when $h(x)=0$.

If $A$ is nilpotent or diagonalizable with real rational eigenvalues, then the controller synthesis problem is decidable.

Linear systems that are completely controllable by each component of the input are called normal in the optimal control literature. It is well known that time-optimal controllers of normal systems have no singular conditions: conditions where the optimal input is undetermined for a finite time interval [6]. In 
fact, according to the Pontryagin's Maximum Principle [13], for a normal linear system, the time-optimal control exists, is unique, and is piecewise constant that taking values on the vertices of the feasible input set. Moreover, the optimal control has a finite number of switchings if the dynamic matrix $A$ has purely real eigenvalues. These results will be crucial in establishing the well-posedness of our models, and the termination of the following controller synthesis procedure.

\section{Controller Synthesis Methodology}

1. Apply Maximum Principle to obtain the saddle solution of optimal $u^{*}, d^{*}$.

2. Construct a hybrid system using the switching logic of optimal $u^{*}, d^{*}$.

3. Perform reachability computations on the constructed hybrid system.

4. Compute the least restrictive controller.

In the next sections, we describe in detail each step of the above procedure.

\section{Differential Games and the Maximum Principle}

In this section, we apply results from differential game theory [210] to formulate the optimal control problem for our controller synthesis methodology. The Hamiltonian for the system (10), is given by $H(x, p, u, d)=p^{T} A x+p^{T} B u+p^{T} E d$. The Hamiltonian satisfies the state and co-state differential equations

$$
\dot{x}=\frac{\partial H}{\partial p}, \quad \dot{p}=-\frac{\partial H}{\partial x}^{T} .
$$

Consider the target set $G=\left\{x \in \mathbb{R}^{n} \mid h(x)<0\right\}$. By setting $p(x, 0)=\frac{\partial h}{\partial x}(x)$, then for every $x \in \partial G, p(x, 0)$ is the outward pointing normal to $\partial G$ at $x$. With this initial condition, the co-state is completely specified by

$$
p(x, 0)=\frac{\partial h}{\partial x}(x), \quad \dot{p}(x, t)=-A^{T} p(x, t) .
$$

Since the goal of the controller is to avoid $G$, the controller tries to maximize the Hamiltonian, while the disturbance tries to minimize it. In this case, the Isaacs condition [2], namely

$$
\max _{u \in U} \min _{d \in D} H(x, p, u, d)=\min _{d \in D} \max _{u \in U} H(x, p, u, d),
$$

is satisfied since the Hamiltonian is separable, i.e. $H(x, p, u, d)=H_{1}(x, p, u)+$ $H_{2}(x, p, d)$. Satisfaction of the Isaacs condition implies that there exists a saddle solution of optimal controls and disturbances $\left(u^{*}, d^{*}\right)$ such that

$$
H\left(x, p, u, d^{*}\right) \leq H\left(x, p, u^{*}, d^{*}\right) \leq H\left(x, p, u^{*}, d\right) .
$$

The saddle solution of optimal controls and disturbances $u^{*}, d^{*}$ satisfies the wellknown Maximum Principle [13]

$$
\left\{\begin{array}{l}
u^{*}\left(x_{0}, t\right) \in \arg \max _{u \in U} p\left(x_{0}, t\right)^{T} B u \\
d^{*}\left(x_{0}, t\right) \in \arg \min _{d \in D} p\left(x_{0}, t\right)^{T} E d .
\end{array}\right.
$$


Equation (15) only constrains the optimal control and disturbance to lie in sets. We will soon see that under the normality condition, these sets are singletons, i.e. the optimal control and disturbance are unique. Starting from an initial $x_{0} \in \partial G$, the input $u^{*}\left(x_{0}, \cdot\right)$ is the best the controller can do to avoid $G$ regardless of the actions of the disturbance, while $d^{*}\left(x_{0}, \cdot\right)$ is the best the disturbance can do to drive the state towards $G$. These controls and disturbances are generally open-loop (as opposed to feedback) policies and are so-called "bang-bang controls" since they switch among the vertices of the set of admissible controls and disturbances. Notice that due to the separability of the Hamiltonian, the problem of computing a saddle solution to the dynamic game reduces to solving two linear optimal control synthesis problems.

Propositions 1 and 2 are fundamental for establishing the well-posedness of our controller synthesis methodology. The proofs are due to Pontryagin [13] and can be found in many optimal control texts, such as [6].

Proposition 1 (Nonsingular Optimal Control and Disturbance). If the linear system (10) is normal with respect to both the control and disturbance, then for any $x_{0} \in \partial G$, the optimal control $u^{*}\left(x_{0}, \cdot\right)$ and disturbance $d^{*}\left(x_{0}, \cdot\right)$ are unique and piece-wise constant taking values on the vertices of $U, D$.

Proposition 2 (Finite Switchings of Optimal Control). If the linear system (10) is normal and $A$ has purely real eigenvalues, then there is a uniform upper bound, independent of $x_{0}$ on the number of switchings of the optimal control $u^{*}\left(x_{0}, \cdot\right)$, and disturbance $d^{*}\left(x_{0}, \cdot\right)$.

\section{Construction of Hybrid System}

The switching policy of the optimal control and disturbance can be naturally abstracted as a hybrid system.

Definition 1 (Hybrid Systems). A hybrid system is a tuple $H=(X, F$, Inv, $R)$ where

- $X=X_{D} \times \mathbb{R}^{m}$ is the state space with $X_{D}=\left\{q_{0}, \ldots, q_{k-1}\right\}$,

$-F: X_{D} \times \mathbb{R}^{m} \rightarrow \mathbb{R}^{m}$ assigns to each discrete location $q \in X_{D}$ a differential equation $\dot{x}=F(q, x)$,

- Inv $: X_{D} \rightarrow 2^{\mathbb{R}^{m}}$ assigns to each discrete location an invariant set $\operatorname{Inv}(q) \subseteq$ $\mathbb{R}^{m}$, and

$-R \subseteq X \times X$ is a relation capturing the discrete transitions .

The elements of $X_{D}$ are the discrete states whereas $x \in \mathbb{R}^{m}$ is the continuous state. Hybrid systems are typically represented as graphs with vertices $X_{D}$, and edges $E$ defined by

$$
E=\left\{\left(q, q^{\prime}\right) \in X_{D} \times X_{D} \mid\left(q, x, q^{\prime}, x^{\prime}\right) \in R \text { for some } x, x^{\prime} \in \mathbb{R}^{m}\right\}
$$


With each edge $e=\left(q, q^{\prime}\right) \in E$ we associate a guard set defined as

$$
\operatorname{Guard}(e)=\left\{x \in \operatorname{Inv}(q) \mid\left(q, x, q^{\prime}, x^{\prime}\right) \in R \text { for some } x^{\prime} \in \mathbb{R}^{m}\right\}
$$

and the set valued reset map

$$
\operatorname{Reset}(e, x)=\left\{x^{\prime} \in \operatorname{Inv}\left(q^{\prime}\right) \mid\left(q, x, q^{\prime}, x^{\prime}\right) \in R\right\} .
$$

Due to switched nature of the optimal control and disturbance, in this paper, it will suffice to assume that for all $e \in E, \operatorname{Reset}(e, x)=x$. Therefore, all reset maps will be the identity map. Furthermore, we do not require the explicit specification of any initial states for our hybrid system.

The solution of the dynamic game played between the control and the disturbance $d$ can be naturally encoded by a hybrid system. The optimal controls and disturbances always lie on the vertices of the admissible set of controls and disturbances $U$ and $D$ which are $n_{u}$ and $n_{d}$ dimensional rectangles. Thus, there are $2^{n_{u}} \cdot 2^{n_{d}}$ possible vector fields associated with the optimal controls and disturbances. We can therefore construct a hybrid system with $2^{n_{u}} \cdot 2^{n_{d}}$ discrete states, one for each possible control/disturbance pair.

We naturally encode the discrete states as a string of boolean numbers of length $n_{u}+n_{d}$. The first $n_{u}$ elements encode the value that the $i$-th component of the optimal control. Similarly the last $n_{d}$ components encode the value of the optimal disturbance. We adopt the convention that 1 stands for the upper bound $\left(u_{i}^{*}=\bar{U}_{i}\right.$ or $\left.d_{i}^{*}=\bar{D}_{i}\right)$, and 0 stands for the lower bound $\left(u_{i}^{*}=\underline{U}_{i}\right.$ or $\left.d_{i}^{*}=\underline{D}_{i}\right)$. For example, in a system with two controls and one disturbance, the discrete state $(0,0,1)$ stands for the case where $u_{1}^{*}=\underline{U}_{1}, u_{2}^{*}=\underline{U}_{2}$, and $d_{1}^{*}=\bar{D}_{1}$. It is therefore clear that the number of discrete states is $2^{n_{u}+n_{d}}$, since $X_{D}$ contains all such boolean strings. According to which is notationally most convenient in the context, we will refer to discrete state $k$ as either $q_{k}$ or the boolean string that represents $k$ in binary. That is, for the example above we may refer to discrete state 5 as either $q_{5}$ or $(1,0,1)$.

Since the optimal control depends on the co-state $p$, the continuous state associated with the hybrid system is actually $(x, p)^{T} \in \mathbb{R}^{2 n}$. The vector field with each discrete state $q_{j}$ then

$$
\left(\begin{array}{c}
\dot{x} \\
\dot{p}
\end{array}\right)=\left(\begin{array}{cc}
A & 0 \\
0 & -A^{T}
\end{array}\right)\left(\begin{array}{c}
x \\
p
\end{array}\right)+\left(\begin{array}{c}
B \\
0
\end{array}\right) u_{q_{j}}+\left(\begin{array}{c}
E \\
0
\end{array}\right) d_{q_{j}},
$$

where $u_{q_{j}} \in \mathbb{R}^{n_{u}}$ and $d_{q_{j}} \in \mathbb{R}^{n_{d}}$ are the constant controls and disturbances associated with discrete state $q_{j}$.

Let $\left(s_{1}, \ldots, s_{n_{u}}, t_{1}, \ldots, t_{n_{d}}\right) \in X_{D}$ where all the $s_{i}$ and $t_{i}$ are either zero or one. Consider the formulas

$$
\begin{aligned}
& I_{i}^{u}(s)=\left\{\begin{array}{l}
p^{T}(-A)^{\beta_{i}(p)} b_{i}>0 \text { if } s=1 \\
p^{T}(-A)^{\beta_{i}(p)} b_{i}<0 \text { if } s=0
\end{array}\right. \\
& I_{i}^{d}(s)=\left\{\begin{array}{l}
p^{T}(-A)^{\varepsilon_{i}(p)} e_{i}<0 \text { if } s=1 \\
p^{T}(-A)^{\varepsilon_{i}(p)} e_{i}>0 \text { if } s=0,
\end{array}\right.
\end{aligned}
$$


where $b_{i}$ and $e_{i}$ are the columns of $B$ and $E$ respectively, and $\beta_{i}(\cdot), \varepsilon_{i}(\cdot)$ are the relative degrees that are now defined.

Definition 2 (Relative Degree). The relative degrees of the $i$-th input and disturbance are functions $\beta_{i}, \varepsilon_{i}: \mathbb{R}^{n} \rightarrow \mathbb{Z}$ defined by:

$$
\begin{aligned}
& \beta_{i}(p)=\left\{\begin{array}{l}
0 \text { if } p^{T} b_{i} \neq 0 \\
1 \text { if } p^{T} b_{i}=0 \wedge p^{T}(-A) b_{i} \neq 0 \\
\vdots \\
j \text { if } \bigwedge_{k=0}^{j-1} p^{T}(-A)^{k} b_{i}=0 \wedge p^{T}(-A)^{j} b_{i} \neq 0
\end{array}\right. \\
& \varepsilon_{i}(p)=\left\{\begin{array}{c}
0 \text { if } p^{T} e_{i} \neq 0 \\
1 \text { if } p^{T} e_{i}=0 \wedge p^{T}(-A) e_{i} \neq 0 \\
\vdots \\
j \text { if } \bigwedge_{k=0}^{j-1} p^{T}(-A)^{k} e_{i}=0 \wedge p^{T}(-A)^{j} e_{i} \neq 0 .
\end{array}\right.
\end{aligned}
$$

The invariant set associated with discrete state $\left(s_{1}, \ldots, s_{n_{u}}, t_{1}, \ldots, t_{n_{d}}\right)$ is simply

$$
\operatorname{Inv}\left(\left(s_{1}, \ldots, s_{n_{u}}, t_{1}, \ldots, t_{n_{d}}\right)\right)=\bigwedge_{i=1}^{n_{u}} I_{i}^{u}\left(s_{i}\right) \wedge \bigwedge_{j=1}^{n_{d}} I_{i}^{d}\left(t_{j}\right)
$$

In other words, the optimal control and disturbance remain the same as long as the signs of all components of $p^{T} B$ and $p^{T} E$ do not change. Proposition 1 ensures that components of $p^{T} B$ and $p^{T} E$ cannot be zero for nontrivial intervals of time, and, furthermore, if some component of $p^{T} B$ or $p^{T} E$ is momentarily zero, the optimal control and disturbance can be uniquely determined by looking at the first nonzero Lie derivative.

Since, in general, the optimal policy can jump from any control/disturbance pair to any other control/disturbance pair, the edge relation $E$ is all of $X_{D} \times X_{D}$. Consider discrete states $\left(s_{1}^{1}, \ldots, s_{n_{u}}^{1}, t_{1}^{1}, \ldots, t_{n_{d}}^{1}\right)$ and $\left(s_{1}^{2}, \ldots, s_{n_{u}}^{2}, t_{1}^{2}, \ldots, t_{n_{d}}^{2}\right)$ and let $J_{u}$ be the set of indices $i$ in $\left\{1, \ldots, n_{u}\right\}$ such that $s_{i}^{1} \neq s_{i}^{2}$. Thus $J_{u}$ contains the indices of all control components that switch optimal policy. Similarly define $J_{d}$. The guard that enables the transition $e$ from $\left(s_{1}^{1}, \ldots, s_{n_{u}}^{1}, t_{1}^{1}, \ldots, t_{n_{d}}^{1}\right)$ to $\left(s_{1}^{2}, \ldots, s_{n_{u}}^{2}, t_{1}^{2}, \ldots, t_{n_{d}}^{2}\right)$ is given by

$$
\operatorname{Guard}(e)=\bigwedge_{i \in J_{u}} I_{i}^{u}\left(\overline{s_{i}}\right) \wedge \bigwedge_{j \in J_{d}} I_{i}^{d}\left(\overline{t_{j}}\right) .
$$

where $\bar{s}$ denotes the boolean complement of $s$.

Notice that for each discrete state, the invariant and the guard depend only on the co-state $p$. Therefore, there are formulas $\operatorname{Inv}_{j}: \mathbb{R}^{n} \rightarrow\{$ TRUE, FALSE $\}$ for $j \in\left\{0, \ldots, 2^{n_{u}+n_{d}}-1\right\}$ such that

$$
\operatorname{Inv}\left(q_{j}\right)=\left\{(x, p)^{T} \in \mathbb{R}^{2 n} \mid \operatorname{Inv}_{j}(p)\right\}
$$

The formulas $\operatorname{Inv}_{j}$ will be used for notational convenience in the reach set computation of the next section. This concludes the specification of the optimal control 


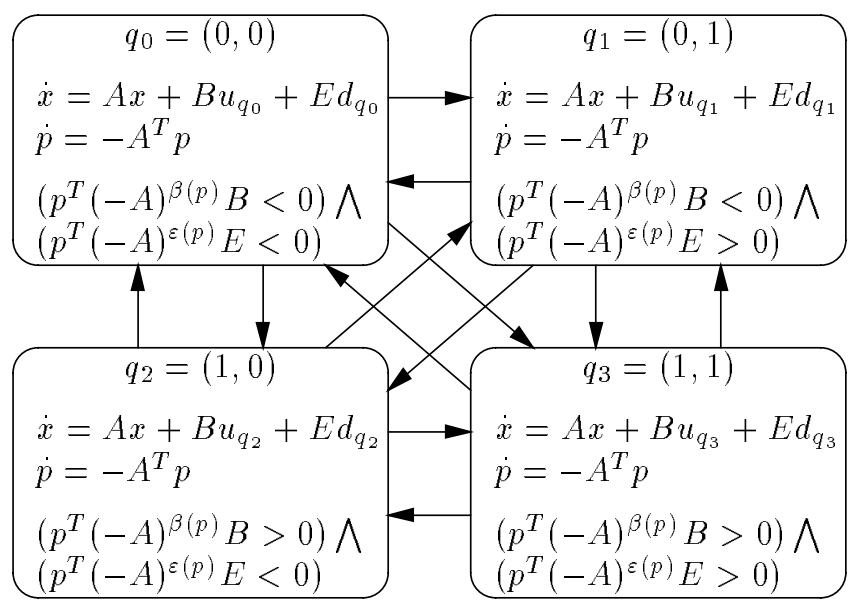

Fig. 1. Natural encoding of game solution as a hybrid system

policy as a hybrid system. Figure 1 shows a block diagram of a hybrid system constructed out of a differential game between one control and one disturbance.

From Propositions 1 and 2 it is straightforward to show that the hybrid system we construct is also well defined in the following sense.

Proposition 3 (Properties of Hybrid System). The hybrid system constructed above is nonblocking, deterministic, and non-Zeno.

The problem of computing the maximal controlled invariant set $W$ has thus been transformed to the problem of computing all states of the hybrid system constructed above that the $x$ component of the continuous state can reach $G$. This reachability computation is the goal of the next section.

\section{Reachability Computation}

For the vector field associated with each discrete state $q_{j}$, we define the predecessor operator Pre $_{j}: 2^{\mathbb{R}^{2 n}} \rightarrow 2^{\mathbb{R}^{2 n}}$. Suppose a set $K \subset \mathbb{R}^{2 n}$ is defined by $K=\left\{(x, p) \in \mathbb{R}^{2 n} \mid P(x, p)\right\}$. Then $\operatorname{Pre}_{j}(K)$ is defined by

$$
\begin{aligned}
\operatorname{Pre}_{j}(K)=\left\{(x, p)^{T} \in \mathbb{R}^{2 n} \mid\right. & \exists y \exists q \exists t: P(y, q) \wedge t \geq 0 \wedge q=e^{-t A^{T}} p \\
& \wedge y=e^{t A} x+\left(\int_{0}^{t} e^{(t-s) A} d s\right)\left(B u_{q_{j}}+E d_{q_{j}}\right) \\
& \left.\wedge \forall s: 0 \leq s \leq t \Rightarrow \operatorname{Inv}_{j}\left(e^{-s A^{T}} p\right)\right\} .
\end{aligned}
$$

An immediate corollary of the main theorem of [9], which is based on the results in [78], is the following:

Proposition 4. Consider a semialgebraic set $K \subset \mathbb{R}^{n}$ and a dynamic system $\dot{x}=A x+b$ where $A \in \mathbb{Q}^{n \times n}, b \in \mathbb{Q}^{n}$. If $A$ is nilpotent or diagonalizable with real rational eigenvalues, then computing the states that can reach $K$ is decidable. 
Proof. Suppose the $K$ is defined by $K=\left\{x \in \mathbb{R}^{n} \mid P(x)\right\}$. By defining

$$
\phi(x, t)=e^{A t} x+\int_{0}^{t} e^{A(t-s)} b d s
$$

we have that the set of states that can reach $K$ is given by $\left\{x \in \mathbb{R}^{n} \mid \exists y \exists t\right.$ : $P(y) \wedge t \geq 0 \wedge y=\phi(x, t)\}$. In order to prove the result, we must show that for each condition on $A$ above, $\phi(x, t)$ can be converted to an equivalent formula in $(\mathbb{R},<,+, \cdot, 0,1)$, which is decidable. If $A$ is nilpotent, then each entry of $e^{A t}$ is polynomial in $t$. Therefore each entry of $\phi(x, t)$ is polynomial in $t$ and hence definable in $(\mathbb{R},<,+, \cdot, 0,1)$. If $A$ is diagonalizable with real eigenvalues then each entry of $e^{A t}$ is a linear combination of the functions $e^{\lambda_{i} t}$ with $\lambda_{i}$ an eigenvalue of $A$. Since the entries of $e^{-A s}$ are linear combinations of $e^{-\lambda_{i} s}$, after integration the entries of $\phi(x, t)$ are linear combinations of $e^{\lambda_{i} t}, e^{-\lambda_{i} t}$. If $\lambda \in \mathbb{Q}$, then by the procedure outlined in [9], $\phi(x, t)$ may be converted into an equivalent formula in $(\mathbb{R},<,+, \cdot, 0,1)$.

An immediate result of Proposition 4 is that the computation of $\operatorname{Pre}_{j}(K)$ is decidable for each discrete state $q_{j}$ if $K$ is a semialgebraic set, and $A$ is either nilpotent, or is diagonalizable with real eigenvalues. Notice that if $K$ is semialgebraic, then so is $\operatorname{Pre}_{j}(K)$.

Now, our goal is to compute all the states of the dynamical game (10) for which the disturbance can drive the state into reach the target set $G$ regardless of the input. In fact, it is only necessary to compute the states that for which the disturbance can drive the state to the "Usable Part" of $G$ :

Definition 3 (Usable Part). The Usable Part (UP) of the target set $G$ is the subset of $\partial G$ for which the disturbance can instantaneously drive the state into $G$ regardless of the control action. Thus UP for the dynamic game (10) and the target set (11) is given by:

$$
U P=\left\{x \in \partial G \mid \forall u \in U \exists d \in D \quad\left(\frac{\partial h(x)}{\partial x}\right)^{T}(A x+B u+E d)<0\right\} .
$$

Since $h(x)$ is a polynomial, then the defining formula (25) for UP is definable in the theory of the reals $(\mathbb{R},<,+, \cdot, 0,1)$ which is known to admit quantifier elimination and be decidable [14]. Therefore computing UP is decidable. Since the hybrid system has the identity as its reset map, any trajectory that enters $G$ if and only if is passes through UP. Now, we need to convert our reachability specification of the linear system (10) into a specification for our abstracted hybrid system. To this end, we define the set

$$
\widetilde{\mathrm{UP}}=\left\{(x, p) \in \mathbb{R}^{2 n} \mid x \in \mathrm{UP}, p=\frac{\partial h(x)}{\partial x}^{T}\right\} .
$$

Define Pre $: 2^{\mathbb{R}^{2 n}} \rightarrow 2^{\mathbb{R}^{2 n}}$ such that for a given $K \subset \mathbb{R}^{2 n}$, Pre $(K)$ is the set of all states of the hybrid system that can reach $K$. It is easily seen that

$$
W^{c}=\left\{x \in \mathbb{R}^{n} \mid \exists p:(x, p) \in \operatorname{Pre}(\widetilde{\mathrm{UP}}) \vee x \in G\right\}
$$


Therefore, the computation of $W$ is decidable if and only if the computation of Pre( $(\widetilde{\mathrm{UP}})$ is decidable.

We now turn to computing Pre( $\widetilde{\mathrm{UP}})$. In general, different states on UP may require different optimal control and disturbance values. We therefore partition $\widetilde{\mathrm{UP}}$ into a disjoint union of subsets according to the optimal controls and disturbances. That is, we partition $\widetilde{\mathrm{UP}}=\bigcup_{q \in Q} S_{q}$, where $Q \subseteq X_{D}$ and the set $S_{q}$ contains those state of $\widetilde{\mathrm{UP}}$ for which the optimal control and disturbance of the are represented by discrete state $q$ of the hybrid system. Since there are only $2^{n_{u}+n_{d}}$ possible optimal controls and disturbances, the partition is finite. Using similar quantifier elimination arguments, it is straightforward to show that the computation of this partition is decidable. Since we have that

$$
\operatorname{Pre}(\widetilde{\mathrm{UP}})=\operatorname{Pre}\left(\bigcup_{q \in Q} S_{q}\right)=\bigcup_{q \in Q} \operatorname{Pre}\left(S_{q}\right),
$$

we can concentrate of computing $\operatorname{Pre}\left(S_{q}\right)$ for a given $q \in Q$. We know that the initial optimal control and disturbance is equal for all initial conditions in $S_{q}$, therefore $S_{q}$ is contained within the same discrete state.

Theorem 2 (Computation of Maximal Controlled Invariant Set). Consider a dynamic game $\dot{x}=A x+B u+E d$ with controls $u \in U \subset \mathbb{R}^{n_{u}}$, disturbances $d \in D \subset \mathbb{R}^{n_{d}}$ and target set $G \subset \mathbb{R}^{n}$ given by $G=\left\{x \in \mathbb{R}^{n} \mid h(x)<0\right\}$. Suppose the system and target set satisfy the following properties:

1. $A \in \mathbb{Q}^{n \times n}, B \in \mathbb{Q}^{n \times n_{u}}, E \in \mathbb{Q}^{n \times n_{d}}$,

2. For each column $b_{i}$ of $B$, the pair $\left(A, b_{i}\right)$ is completely controllable,

3. For each column $e_{i}$ of $E$, the pair $\left(A, e_{i}\right)$ is completely controllable,

4. The feasible sets of controls $U$ and disturbances $D$ are compact rectangles with rational vertices,

5. $h \in \mathbb{Q}\left[x_{1}, x_{2}, \ldots, x_{n}\right]$ and $\frac{\partial h}{\partial x}(x) \neq 0$ when $h(x)=0$.

If $A$ is nilpotent or diagonalizable with real rational eigenvalues, then the computation of the maximal controlled invariant set $W$ is decidable.

Proof. Due to our partition in equation (28), we have

$$
\begin{aligned}
W^{c} & =\left\{x \in \mathbb{R}^{n} \mid \exists p:(x, p) \in \operatorname{Pre}(\widetilde{\mathrm{UP}})\right\} \cup G \\
& =\bigcup_{q \in Q}\left\{x \in \mathbb{R}^{n} \mid \exists p:(x, p) \in \operatorname{Pre}\left(S_{q}\right)\right\} \cup G
\end{aligned}
$$

where each of the above steps is decidable. Thus it suffices to show that for a given $q \in Q$ computing $\operatorname{Pre}\left(S_{q}\right)$ is decidable.

Due to Proposition [3] we need not worry about any pathologies in the Pre computation. Since, in each discrete state, the optimal input and disturbance are constant we apply Proposition 4 to decidably compute the set of states that can reach $S_{q}$ for that particular combination of control/disturbance pair. However, the optimal control or disturbance may change and a discrete transition may be 
taken. The predecessor operator of the discrete jumps is trivial since the reset map of our jumps is the identity map.

Now, if the matrix $A$ has real eigenvalues, then due to Proposition 2 after a finite number of switchings, uniformly in $(x, p)^{T} \in S_{q}$, there are no more switches and we can use Proposition 4 one last time. Therefore, the algorithm terminates after a finite number of steps.

\section{Least Restrictive Controller}

Our goal in this section is to compute the least restrictive controller that renders the maximum controlled invariant set $W$ invariant. The result of the previous section is that $W$ is definable in $(\mathbb{R},<,+, \cdot, 0,1)$ which is decidable 14. Since $(\mathbb{R},<,+, \cdot, 0,1)$ admits quantifier elimination, we may compute a quantifier-free formula $\psi$ such that $W=\left\{x \in \mathbb{R}^{n} \mid \psi(x)\right\}$. The quantifier elimination that is required in this procedure can be done by the computer logic software systems REDLOG [5] or QEPCAD [4]. The defining formula of the set $W$ may be converted to the so-called disjunctive normal form to yield:

$$
W=\left\{x \in \mathbb{R}^{n} \mid \bigvee_{j=1}^{L}\left(\bigwedge_{k=1}^{M_{j}} f_{j_{k}}(x) m_{j_{k}} 0\right)\right\}
$$

where $f_{j_{k}} \in \mathbb{Q}\left[x_{1}, \ldots, x_{n}\right]$ and $m_{j_{k}} \in\{<, \leq,<, \geq,=, \neq\}$.

Since the least restrictive controller specifies a control action only on the boundary of $W$, our first task is to compute the boundary of $W, \partial W$. We will need the following lemma from [17].

Lemma 1. If $W \subset \mathbb{R}^{n}$ is definable in a decidable theory, then so is the closure $\bar{W}$, the interior $W^{o}$, and the boundary $\partial W$.

Proof. For a set $W=\left\{x \in \mathbb{R}^{n} \mid \psi(x)\right\}$, the sets $\bar{W}$ and $W^{o}$ are given by

$$
\begin{aligned}
& \bar{W}=\left\{x \in \mathbb{R}^{n} \mid \forall\left(y_{1}, \ldots, y_{n}\right) \forall\left(z_{1}, \ldots, z_{n}\right):\left[\wedge_{i=1}^{n} y_{i}<x_{i}<z_{i} \Rightarrow\right.\right. \\
& \left.\left.\exists\left(w_{1}, \ldots, w_{n}\right): \wedge_{i=1}^{n} y_{i}<w_{i}<z_{i} \wedge \psi(w)\right]\right\} \\
& W^{o}=\left\{x \in \mathbb{R}^{n} \mid \exists\left(y_{1}, \ldots, y_{n}\right) \exists\left(z_{1}, \ldots, z_{n}\right):\left[\wedge_{i=1}^{n} y_{i}<x_{i}<z_{i} \wedge\right.\right. \\
& \left.\left.\forall\left(w_{1}, \ldots, w_{n}\right): \wedge_{i=1}^{n} y_{i}<w_{i}<z_{i} \Rightarrow \psi(w)\right]\right\}
\end{aligned}
$$

where we use the shorthand notation $(\alpha \Rightarrow \beta) \equiv(\neg \alpha \vee \beta)$. The expressions (32) and (33) are simply the definitions of closure and interior in the usual topology of $\mathbb{R}^{n}$. Let the defining formulas for $\bar{W}, W^{o}$ be $\bar{\psi}, \psi^{o}$ respectively. Then the defining formula for $\partial W$ is simply $\partial \psi \equiv \bar{\psi} \wedge\left(\neg \psi^{o}\right)$. Clearly if $\psi$ is defined in a theory which admits quantifier elimination, then so are $\bar{\psi}, \psi^{o}$, and $\partial \psi$.

From the Lemma 1 we have that $\partial W$ may be defined by a quantifier-free formula

$$
\partial W=\left\{x \in \mathbb{R}^{n} \mid \partial \psi(x)\right\} .
$$


Since the least restrictive controller only specifies a control action on $\partial W$, then $g: \mathbb{R}^{n} \rightarrow 2^{U}$ must be of the form

$$
g(x)=\{u \in U \mid \partial \psi(x) \Rightarrow \phi(x, u)\}
$$

where $\phi(x, u)$ is a formula to be described below.

Denote $W=\bigcup_{j=1}^{L}\left(\bigcap_{k=1}^{M_{j}} W_{j_{k}}\right)$, and consider the least restrictive controller for a single polynomial constraint $W_{j_{k}}=\left\{x \in \mathbb{R}^{n} \mid f_{j_{k}}(x) m_{j_{k}} 0\right\}$. For this polynomial constraint, we define the formula

$$
\phi_{j_{k}}(x, u) \equiv\left(\left(f_{j_{k}}(x)=0\right) \Rightarrow \forall d \in D:{\frac{\partial f_{j_{k}}(x)}{\partial x}}^{T}(A x+B u+E d) m_{j_{k}} 0\right) .
$$

Using equation (36), it is direct to see that the least restrictive controller that renders $W_{j_{k}}$ invariant is given by $g_{j_{k}}(x)=\left\{u \in U \mid \phi_{j_{k}}(x, u)\right\}$. This least restrictive controller is simply a re-writing of equation (9) in terms of a decidable formula. Now, the least restrictive controller for $W=\bigcup_{j=1}^{L}\left(\bigcap_{k=1}^{M_{j}} W_{j_{k}}\right)$ must be satisfy each of the of the simpler constraints, and hence is given by the following.

Theorem 3 (Least Restrictive Controller). For the differential game $\dot{x}=$ $A x+B u+E d$, the least restrictive controller $g: \mathbb{R}^{n} \rightarrow 2^{U}$ that renders the set $W=\left\{x \in \mathbb{R}^{n} \mid \bigvee_{j=1}^{L}\left(\bigwedge_{k=1}^{M_{j}} f_{j_{k}}(x) m_{j_{k}} 0\right)\right\}$ invariant is given by

$$
g(x)=\left\{u \in U \mid \partial \psi(x) \Rightarrow \bigvee_{j=1}^{L}\left(\bigwedge_{k=1}^{M_{j}} \phi_{j_{k}}(x, u)\right)\right\}
$$

where $\partial \psi$ is the defining formula of $\partial W$ and $\phi_{j_{k}}(x, u)$ is given by equation (36). If $W$ is definable in a decidable theory, then so is $g(x)$.

Therefore, Theorems 2 and 3 collectively result in Theorem 1 .

\section{Conclusions}

In this paper we have shown that controller synthesis for classes of linear systems with polynomial reachability specifications is decidable. In further research, we will extend the target set $G$ to a semialgebraic set, investigate conditions for semi-decidability in the absence of the normality condition, and extend the continuous decidability results to semidecidability results for classes of linear hybrid systems. In the case of purely imaginary eigenvalues, the problem becomes quickly undecidable unless one remains in a compact region of the state space. The observation along with the results of this paper have a clear and natural connection with o-minimal theories of the reals [7 17], which will explored in future research.

\section{Acknowledgments}

This research has been supported by DARPA under grant F33615-98-C-3614, and DARPA/NASA grant NAG2-1214. 


\section{References}

1. E. Asarin, O. Maler, and A. Pnueli. Symbolic controller synthesis for discrete and timed systems. In P. Antsaklis, W. Kohn, A. Nerode, and S. Sastry, editors, Hybrid Systems II, volume 999 of Lecture Notes in Computer Science. SpringerVerlag, 1995.

2. T. Başar and G.J. Olsder. Dynamic Noncooperative Game Theory. Academic Press, 2nd edition, 1995.

3. A. Church. Logic, arithmetic, and automata. In Proceedings of the International Congress of Mathematics, pages 23-35, 1962.

4. G.E. Collins and H. Hong. Partial cylindrical algebraic decomposition for quantifier elimination. Journal of Symbolic Computation, 12:299-328, September 1991.

5. A. Dolzman and T. Sturm. REDLOG : Computer algebra meets computer logic. ACM SIGSAM Bulletin, 31(2):2-9, June 1997.

6. D.E. Kirk. Optimal Control Theory, An Introduction. Prentice Hall, 1970.

7. G. Lafferriere, G. J. Pappas, and S. Sastry. O-minimal hybrid systems. Mathematics of Control, Signals, and Systems. To appear.

8. G. Lafferriere, G. J. Pappas, and S. Yovine. A new class of decidable hybrid systems. In Hybrid Systems : Computation and Control, volume 1569 of Lecture Notes in Computer Science, pages 137-151. Springer Verlag, 1999.

9. G. Lafferriere, G. J. Pappas, and Sergio Yovine. Reachability computation for linear hybrid systems. In Proceedings of the 14th IFAC World Congress, volume E, pages 7-12, Beijing, P.R. China, July 1999.

10. J. Lewin. Differential Games. Springer Verlag, 1994.

11. J. Lygeros, D.N. Godbole, and S. Sastry. Verified hybrid controllers for automated vehicles. IEEE Transactions on Automatic Control, 43(4):522-539, April 1998.

12. J. Lygeros, C. Tomlin, and S.S. Sastry. Controllers for reachability specifications for hybrid systems. Automatica, 35(3):349-370, March 1999.

13. L.S. Pontryagin, V. Boltyanskii, R. Gamkrelidze, and E. Mischenko. The Mathematical Theory of Optimal Processes. John Wiley \& Sons, 1962.

14. A. Tarski. A decision method for elementary algebra and geometry. University of California Press, second edition, 1951.

15. W. Thomas. On the synthesis of strategies in infinite games. In Ernst W. Mayr and Claude Puech, editors, Proceedings of STACS 95, Volume 900 of LNCS, pages 1-13. Springer Verlag, Munich, 1995.

16. C. Tomlin, G. J. Pappas, and S. Sastry. Conflict resolution for air traffic management : A study in muti-agent hybrid systems. IEEE Transactions on Automatic Control, 43(4):509-521, April 1998.

17. L. van den Dries. Tame Topology and o-minimal structures. Cambridge University Press, 1998.

18. H. Wong-Toi. The synthesis of controllers for linear hybrid automata. In Proceedings of the 36th IEEE Conference on Decision and Control, San Diego, CA, December 1997. 